QUARTERLY OF APPLIED MATHEMATICS

VOLUME LXIV, NUMBER 4

DECEMBER 2006, PAGES 605-615

S $0033-569 X(06) 01018-6$

Article electronically published on November 13, 2006

\title{
ASYMMETRIC EQUILIBRIUM CONFIGURATIONS OF HYPERELASTIC CYLINDRICAL BODIES UNDER SYMMETRIC DEAD LOADS
}

\author{
BY
}

\author{
ANGELO MARCELLO TARANTINO
}

\begin{abstract}
Homogeneous deformations provided by the nonlinear equilibrium problem of symmetrically loaded isotropic hyperelastic cylindrical bodies are investigated. Depending on the form of the stored energy function, the problem considered may admit asymmetric solutions, besides the expected symmetric solutions. For general compressible materials, the mathematical condition allowing the assessment of these asymmetric solutions, which describe the global path of equilibrium branches, is given. Explicit expressions for evaluating critical loads and bifurcation points are derived. Results and basic relations obtained for general isotropic materials are then specialized for a MooneyRivlin and a neo-Hookean material. A broad numerical analysis is performed and the qualitatively more interesting asymmetric equilibrium branches are shown. The influence of the constitutive parameters is discussed, and, using the energy criterion, a number of considerations are carried out concerning the stability of the equilibrium solutions.
\end{abstract}

1. Introduction. Nonlinearities in finite elasticity often lead to multiple equilibrium configurations and, under some circumstances, even in a symmetric layout it is possible to observe an unexpected lack of symmetry of the deformation. A typical experimental behavior of this kind was evidenced by Treloar [1]. In a biaxial stretching test, Treloar stretched a square rubber membrane by equal in-plane biaxial loads applied perpendicular to the membrane edges, and he observed that, when the load exceeds a certain critical value, the membrane can be in equilibrium with unequal stable in-plane principal stretches. Namely, during the deformation process, a homogeneous shape-change mode of bifurcation (square to parallelogram) can occur.

Even from a theoretical point of view, the problem of the existence of asymmetric equilibrium solutions generated by symmetric loads has received great attention. Kearsley [2], analyzed membranes composed of incompressible Mooney-Rivlin material, finding both symmetric and asymmetric solutions. MacSithigh 3 studied the same problem

Received December 8, 2004.

2000 Mathematics Subject Classification. Primary 73G05, 73G10, 73 H05.

Key words and phrases. Finite elasticity, bifurcation, and stability.

Dipartimento di Ingegneria Meccanica e Civile, Università degli Studi di Modena e Reggio Emilia, via Vignolese $905-41100$ Modena, Italy. 
using a minimum energy approach. In order to study the stability of post-bifurcation paths, Ogden [4] considered a local incremental bifurcation mode by looking for nontrivial solutions to the equations of small deformations superposed on finite deformations. Chen [5, 6] found conditions for the stability of equilibria in an arbitrary isotropic, incompressible material, demonstrating that the same equilibrium states are at most neutrally stable. More recently, some constitutive branching analyses, which show how bifurcation depends on the form of the stored energy function, have been proposed by Haslach [7] and Tarantino [8. In 8, the equations governing the global development of the asymmetric equilibrium branches for incompressible isotropic material have been derived.

The corresponding plane deformation problem was studied again by Ogden [9], 10]. In particular, Ogden gave conditions, based on the local invertibility of the elastic stressdeformation relations, to have a bifurcation from the primary path of equal stretch equilibria.

In the present work, the symmetry-breaking phenomenon observed by Treloar is studied, considering general compressible isotropic cylindrical bodies subject to in-plane equibiaxial dead-load tractions. The main purpose is to extend the analysis investigating symmetric and asymmetric solutions admitted by the nonlinear equilibrium problem. Emphasis is placed on the search of equilibrium configurations characterized by lack of uniqueness and on the pertinent bifurcation phenomena.

In Section 2 the finite plane deformation problem for a compressible, isotropic and hyperelastic material is reported. In Section 3 the mathematical condition, in terms of principal deformation invariants, for the computation of asymmetric solutions within the class of homogeneous deformations is derived. Moreover, expressions for the evaluation of critical loads and of the corresponding bifurcation points are obtained. Results and basic equations derived for general isotropic materials are then specialized in Section 4 for a compressible Mooney-Rivlin material and in Section 5 for a compressible neoHookean material. For different combinations of material parameters, explicit branches of asymmetric equilibria are computed and the qualitatively more significant situations are discussed and reported through a series of plots. Using the energy criterion, some stability considerations about the equilibrium solutions are brought forward.

2. The boundary-value problem. In this work we consider homogeneous, hyperelastic and isotropic cylindrical bodies, under the plane deformation condition. Rectangular Cartesian material coordinates $\left(x_{1}, x_{2}, x_{3}\right)$ are chosen to describe a body whose undeformed configuration is the closure of the regular cylindrical domain $\mathrm{B}=\left\{\boldsymbol{x} \mid\left(x_{1}, x_{2}\right) \in\right.$ $\left.\Omega, 0<x_{3}<L\right\}$ of the three-dimensional Euclidean space E. The deformed configuration is given by the deformation $\varphi: \overline{\mathrm{B}} \rightarrow \mathrm{V}$, and, hereinafter, $\mathbf{F}: \overline{\mathrm{B}} \rightarrow \mathcal{L}_{\text {in }}{ }^{+}$indicates the deformation gradient $\mathbf{D} \varphi$ 目

\footnotetext{
${ }^{1} \mathrm{~V}$ is the vector space associated with $\mathrm{E}$ and $\mathcal{L i n}^{+}$is the set of all the (second-order) tensors with positive determinant.
} 
For the cylindrical body $\overline{\mathrm{B}}$, a state of plane deformation parallel to the plane $\Omega\left(x_{1}, x_{2}\right)$ is characterized by 2

$$
\varphi_{\alpha}=\hat{\varphi}_{\alpha}\left(x_{1}, x_{2}\right) \text { and } \varphi_{3}=x_{3} .
$$

Thus, the components of the deformation gradient $\mathbf{F}$ are independent of $x_{3}$ and found to be

$$
F_{\alpha \beta}=\varphi_{\alpha, \beta}\left(x_{1}, x_{2}\right), \quad F_{\alpha 3}=F_{3 \gamma}=0, \quad F_{33}=1 .
$$

Using (11) and denoting by $w=\hat{w}\left(I_{1}, I_{2}, I_{3}\right)$ the stored energy function 3 , where the variables

$$
I_{1}=\lambda_{1}^{2}+\lambda_{2}^{2}+\lambda_{3}^{2}, \quad I_{2}=\lambda_{1}^{2} \lambda_{2}^{2}+\lambda_{1}^{2} \lambda_{3}^{2}+\lambda_{2}^{2} \lambda_{3}^{2}, \quad I_{3}=\delta^{2}=\lambda_{1}^{2} \lambda_{2}^{2} \lambda_{3}^{2},
$$

are the principal invariants of the left Cauchy-Green deformation tensor $\mathbf{B}=\mathbf{F F}^{T}$ and $\lambda_{i}$ are the principal stretches, the nonvanishing components of the Piola-Kirchhoff stress tensor $\mathbf{T}_{\mathbf{R}}$ assume the following form $\left(T_{R \alpha 3}=T_{R 3 \beta}=0\right)$ :

$$
\begin{gathered}
T_{R \alpha \beta}=\gamma_{0} F_{\alpha \beta}^{-T}+\gamma_{1} F_{\alpha \beta}, \\
T_{R 33}=2 w_{1}+2 h w_{2}+2 w_{3} j^{2},
\end{gathered}
$$

where

$$
\gamma_{0}\left(I_{1}, I_{2}, I_{3}\right)=2 j^{2}\left(w_{2}+w_{3}\right), \quad \gamma_{1}\left(I_{1}, I_{2}, I_{3}\right)=2\left(w_{1}+w_{2}\right),
$$

with $j=\lambda_{1} \lambda_{2}, h=\lambda_{1}^{2}+\lambda_{2}^{2}$ and $w_{i}=\partial w / \partial I_{i}$.

The boundary-value problem may now be formulated by considering a cylindrical body with a unit square cross section $\Omega=\left\{\left(x_{1}, x_{2}\right):\left|x_{\alpha}\right|<1 / 2\right\}$, stretched and maintained in equilibrium by tensile surface forces applied uniformly and orthogonally to the four sides. We restrict our attention to dead loads, namely to the simplest kind of applied forces characterized by densities per unit area in the reference configuration that do not depend on the deformation. The boundary-value problem, therefore, consists in finding the solutions $\varphi_{\alpha}$ of the equilibrium equations (in the absence of the body forces)

$$
T_{R \alpha \beta, \beta}=0, \quad \text { for all } x_{\alpha} \in \Omega,
$$

supplemented by the boundary conditions

$$
T_{R \alpha \beta} n_{\beta}=s n_{\alpha}, \quad \text { for }\left|x_{\alpha}\right|=1 / 2,
$$

where $s$ is an assigned positive constant and $n_{\alpha}$ are the components of the outward unit normal to $\partial \Omega$. Solutions to the above problem are sought in the class of homogeneous deformations, namely in the set of deformations with constant deformation gradient, with special attention to those equilibrium configurations characterized by lack of uniqueness.

\footnotetext{
${ }^{2}$ In the sequel, the range of Greek indices is $\{1,2\}$ and that of Latin indices is $\{1,2,3\}$.

${ }^{3} \mathrm{We}$ assume that the stored energy function is smooth enough, bounded below and satisfies the growth conditions [5], 14]: $w(\mathbf{F}) \rightarrow \infty$ as both $\operatorname{det} \mathbf{F} \rightarrow 0^{+}$and $\|\mathbf{F}\| \rightarrow \infty$. These hypotheses are crucial for the existence of (at least) a homogeneous solution.
} 
3. Symmetric and asymmetric solutions. A homogeneous deformation trivially satisfies the field equations (5) and produces a constant stress field that must fulfill the boundary conditions (6). Introducing (3a) into (6), the following set of equations is obtained:

$$
\begin{aligned}
\frac{\gamma_{0}}{j} \varphi_{2,2}+\gamma_{1} \varphi_{1,1} & =s, & -\frac{\gamma_{0}}{j} \varphi_{2,1}+\gamma_{1} \varphi_{1,2} & =0, \\
-\frac{\gamma_{0}}{j} \varphi_{1,2}+\gamma_{1} \varphi_{2,1} & =0, & \frac{\gamma_{0}}{j} \varphi_{1,1}+\gamma_{1} \varphi_{2,2} & =s .
\end{aligned}
$$

With the polar decomposition of the deformation gradient $F_{\alpha \beta}$, the deformation may be regarded as resulting from two (constant) stretchings $\left(\lambda_{1}, \lambda_{2}\right)$ along the principal axes parallel to the plane $\Omega$ (pure deformation), followed by a rigid-body rotation (angle $\theta$ ) of these axes around the principal axis $x_{3}$ normal to $\Omega$. Unlike the case studied in 8 , the orientation of the principal axes is kept unspecified. Therefore, denoting by $\phi$ the angle that the first principal axis forms with the $x_{1}$-axis, the in-plane components of the deformation gradient are expressed as

$$
[F]_{\alpha \beta}=\left[\begin{array}{cc}
\cos \theta & \sin \theta \\
-\sin \theta & \cos \theta
\end{array}\right]\left[\begin{array}{cc}
\cos \phi & \sin \phi \\
-\sin \phi & \cos \phi
\end{array}\right]\left[\begin{array}{cc}
\lambda_{1} & 0 \\
0 & \lambda_{2}
\end{array}\right]\left[\begin{array}{cc}
\cos \phi & -\sin \phi \\
\sin \phi & \cos \phi
\end{array}\right],
$$

and the system (7) can be rewritten as follows:

$$
\begin{aligned}
\left(\gamma_{1}-\frac{\gamma_{0}}{j}\right)\left(\lambda_{1}-\lambda_{2}\right) \cos (2 \phi+\theta) & =0, \\
\left(\gamma_{1}-\frac{\gamma_{0}}{j}\right)\left(\lambda_{1}-\lambda_{2}\right) \sin (2 \phi+\theta) & =0, \\
\left(\gamma_{1}+\frac{\gamma_{0}}{j}\right)\left(\lambda_{1}+\lambda_{2}\right) \cos \theta & =2 s, \\
\left(\gamma_{1}+\frac{\gamma_{0}}{j}\right)\left(\lambda_{1}+\lambda_{2}\right) \sin \theta & =0 .
\end{aligned}
$$

As suggested by (8c), to have bounded values of the stretches, the quantity $\left(\gamma_{1}+\frac{\gamma_{0}}{j}\right)$ must be different from zero. Since the stretches are strictly positive scalars, from $8 \mathrm{dd}$, we then have $\sin \theta=0$, that is $\theta=k \pi$, with $k=0, \pm 1, \pm 2, \ldots$ The body may thus undergo rotations that are multiples of $180^{\circ}$ about the principal axis $x_{3}$ parallel to the generators of B. For odd values of $k$, the tensile dead loads are converted to compressive forces. Such an inversion of sign is allowed by the mathematical idealization of dead loading, according to which the forces preserve their orientation during rotation. Equation (8c), with $\cos k \pi= \pm 1$, provides a relation which links the intensity of the external loads with the principal stretches. Since the two trigonometric functions $\sin (2 \phi+k \pi)$ and $\cos (2 \phi+k \pi)$ cannot vanish simultaneously, equations (8a) and (8b) supply two classes of solutions:

$$
\begin{gathered}
\lambda_{1}-\lambda_{2}=0 \\
\gamma_{1}-\frac{\gamma_{0}}{j}=0 .
\end{gathered}
$$


For both sets of solutions, the angle $\phi$ remains undetermined, as a result of the complete symmetry of the problem.

Equation (9) represents the symmetric solutions. The sought homogeneous deformations transform (homothetically) the unit square cross section $\Omega$ of the undeformed cylindrical body into another square. In particular, the strain ellipse degenerates into a circle and every direction is principal.

On the other hand, Equation (10) constitutes a condition, in terms of principal invariants, which allows unequal values of the principal stretches (asymmetric solutions, $\lambda_{1} \neq \lambda_{2}$ ). In this case, given the arbitrariness of the angle $\phi$, the initial square cross section is transformed into a parallelogram.

The equilibrium problem admits (at least) one solution of the first class, whereas the existence of other solutions belonging to the second class depends on the specific form of the stored energy function.

Through (4), the condition (10) for a general compressible material can be expressed by

$$
w_{1}+(1-j) w_{2}-j w_{3}=0 .
$$

From a mechanical point of view, the more interesting roots of this equation, if they exist, are obviously the asymmetric ones. In this circumstance, applying (11), it is possible to obtain the global development of asymmetric equilibrium branches. With (4), (8c) gives the intensity of the external loads needed to produce the homogeneous deformation with principal stretches $\lambda_{1}$ and $\lambda_{2}$ :

$$
s= \pm\left(\lambda_{1}+\lambda_{2}\right)\left[w_{1}+(1+j) w_{2}+j w_{3}\right] .
$$

In particular, introducing (11) into (12), the intensity of the external loads along any asymmetric equilibrium branch can be evaluated. In this case, (12) reduces to

$$
s= \pm 2\left(\lambda_{1}+\lambda_{2}\right)\left[w_{1}+w_{2}\right] .
$$

At a bifurcation point $\lambda_{1}=\lambda_{2}=\lambda_{C R}$ and the relations (11) and (13) become

$$
\begin{gathered}
w_{1}+\left(1-\lambda_{C R}^{2}\right) w_{2}-\lambda_{C R}^{2} w_{3}=0, \\
s_{C R}= \pm 4 \lambda_{C R}\left[w_{1}+w_{2}\right],
\end{gathered}
$$

where $w_{i}$ now depend on $\lambda_{C R}$.

4. Application to compressible Mooney-Rivlin materials. The stored energy function of compressible Mooney-Rivlin materials has the following form [11:

$$
w=a I_{1}+b I_{2}+G(\delta),
$$

with $a>0$ and $b>0$. The following expression, proposed by Ciarlet and Geymonat [15, is chosen for the function $G(\delta)$ :

$$
G(\delta)=c \delta^{2}-d \ln \delta
$$

with $c>0$ and $d>0$. 


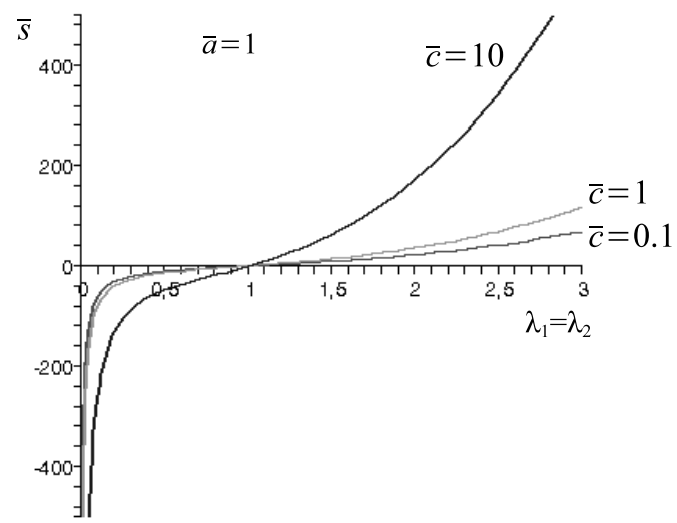

FIG. 1. Plot of the external load versus the in-plane stretch $\lambda_{1}=\lambda_{2}$

Through (16) and (17), the above relations (11) and (12) furnish

$$
\begin{gathered}
-2(a+b) \lambda_{1} \lambda_{2}+2(b+c) \lambda_{1}^{2} \lambda_{2}^{2}-d=0, \\
s= \pm \frac{1}{2} \frac{\lambda_{1}+\lambda_{2}}{\lambda_{1} \lambda_{2}}\left\{2(a+b) \lambda_{1} \lambda_{2}+2(b+c) \lambda_{1}^{2} \lambda_{2}^{2}-d\right\} .
\end{gathered}
$$

Depending on the numerical values of the four material constants, (18) may provide asymmetric solutions. To simplify the parametric analysis, (18) and (19) are rewritten in dimensionless form dividing through by the constant $b(\neq 0)$ :

$$
\begin{gathered}
(\bar{a}+\bar{c}+2)+(\bar{a}+1) \lambda_{1} \lambda_{2}-(\bar{c}+1) \lambda_{1}^{2} \lambda_{2}^{2}=0, \\
\bar{s}= \pm \frac{\lambda_{1}+\lambda_{2}}{\lambda_{1} \lambda_{2}}\left\{-(\bar{a}+\bar{c}+2)+(\bar{a}+1) \lambda_{1} \lambda_{2}+(\bar{c}+1) \lambda_{1}^{2} \lambda_{2}^{2}\right\},
\end{gathered}
$$

where $\bar{a}=a / b, \bar{c}=c / b$ and $\bar{s}=s / b$. To have vanishing stresses when $\lambda_{1}=\lambda_{2}=1$, the dimensionless constants of (20) and (21) have been normalized using the following relation:

$$
\bar{d}=d / b=2(\bar{a}+\bar{c}+2) .
$$

In order to show a typical stress-deformation relation for a cylindrical body composed of compressible Mooney-Rivlin material, Equation (21) is plotted in Figure 1, for the special case $\lambda_{1}=\lambda_{2}$ (primary path), choosing $\bar{a}=1$ and the following three values for $\bar{c}: 0.1,1$ and 10 .

Having selected the two ratios $\bar{a}$ and $\bar{c}$, the implicit Equation (20) provides the branches of the asymmetric equilibrium, along which the initial square cross section of the cylindrical body is deformed into a parallelogram. Taking into account the whole range of values for the two material parameters, equilibrium solutions exhibit qualitative properties that can be described by means of diagrams like that shown in Figure 2, Such a diagram is plotted for a specific value of $\bar{a}$, and each single curve refers to a particular value of $\bar{c}$. A single point $\left(\lambda_{1}, \lambda_{2}\right)$ of each curve represents an equilibrium configuration of the body 


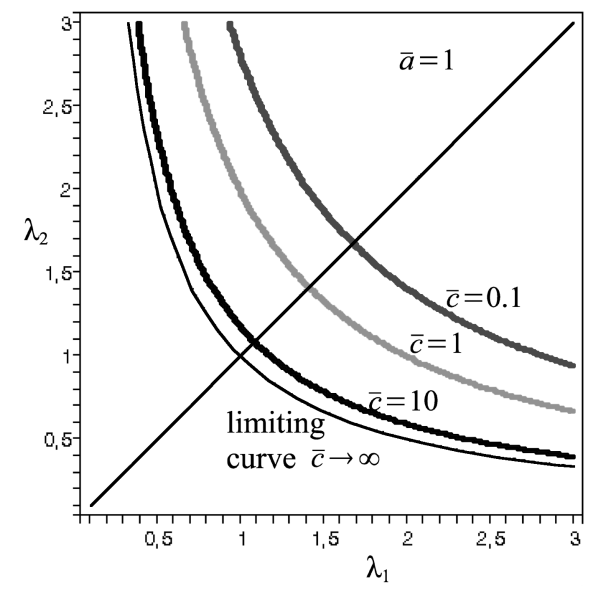

Fig. 2. Branches of asymmetric equilibria on the $\left(\lambda_{1}, \lambda_{2}\right)$-plane, with $\bar{a}=1$

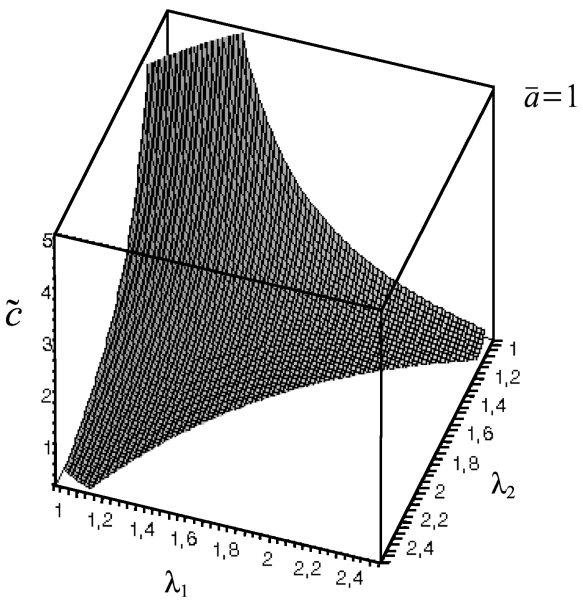

FIG. 3. Plot of surface $\tilde{c}$, with $\bar{a}=1$

under a prescribed load. Changing the loading parameter $\bar{s}$, the point moves along the same curve and, in general, different equilibrium points of a single curve may correspond to the same value of $\bar{s}$. The bisector $\lambda_{1}=\lambda_{2}$, which represents the universal symmetric solutions, is also reported.

All curves describing the branches of asymmetric equilibria have the same hyperbolalike form ( $c f$. Figure 2). Increasing $\bar{c}$, these hyperbolas translate toward the limiting curve $(\bar{c} \rightarrow \infty)$

$$
\lambda_{1}^{2} \lambda_{2}^{2}-1=0
$$

which is plotted in Figure 2 with a dashed line. Note that such a limiting curve describes the equilibrium solutions for incompressible materials. To better understand the modifications that the branches of the asymmetric equilibria undergo when the material 


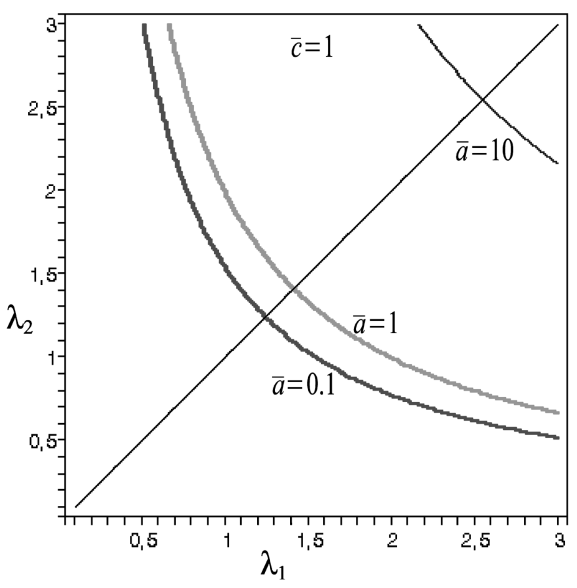

FIG. 4. Branches of asymmetric equilibria on the $\left(\lambda_{1}, \lambda_{2}\right)$-plane, with $\bar{c}=1$

parameter $\bar{c}$ is changed, 3D graphics may be employed such as in Figure 3 In this figure, the surface, obtained from Equation (20) keeping $\bar{a}$ fixed,

$$
\tilde{c}\left(\lambda_{1}, \lambda_{2} ; \bar{a}\right)=\frac{\bar{a}+2+(\bar{a}+1) \lambda_{1} \lambda_{2}-\lambda_{1}^{2} \lambda_{2}^{2}}{\lambda_{1}^{2} \lambda_{2}^{2}-1}
$$

is plotted. The curves of Figure 2 can be obtained as contour lines slicing the surface $\tilde{c}$ through horizontal planes. Since the surface $\tilde{c}$ is a monotonically decreasing function, its intersection with horizontal planes cannot yield more than a single open-curve solution, for each pair of assigned parameters $\bar{a}$ and $\bar{c}$.

The influence of the material parameter $\bar{a}$ is shown in Figure 4, where the branches of asymmetric equilibria are drawn for a fixed value of the parameter $\bar{c}$ : as $\bar{a}$ increases, the curves drift away from the origin.

The bifurcation points, that is, the intersections of asymmetric equilibrium branches with the bisector, can be found by sectioning the surface $\tilde{c}$ with the vertical plane $\lambda_{1}=\lambda_{2}$. In this way, an equation that relates the material parameters and the critical stretch $\lambda_{C R}$ is obtained:

$$
\tilde{c}\left(\lambda_{C R} ; \bar{a}\right)=\frac{\bar{a}+2+(\bar{a}+1) \lambda_{C R}^{2}-\lambda_{C R}^{4}}{\lambda_{C R}^{4}-1} .
$$

Equation (25) is plotted in Figure 5 for $\bar{a}=0.1,1$ and 10 .

According to the energy criterion of stability analysis, an equilibrium configuration under assigned dead loads is said to be (locally) stable if the corresponding deformation is a (relative) minimizer of the total energy. Although a complete stability analysis is not performed in this section, contour lines for the specific energy $e\left(\lambda_{1}, \lambda_{2}\right)=\tilde{w}\left(\lambda_{1}, \lambda_{2}\right)-\mathbf{T}_{\mathbf{R}} \cdot \mathbf{F}$ are shown in the neighborhoods of the asymmetric equilibrium solutions 4 These contour lines are plotted in Figure 6 for the case with $\bar{a}=1$ and $\bar{c}=1$. The location of minimum points on the first segment of the bisector states that the symmetric solutions are initially stable. Increasing the loading parameter $s$, the bifurcation point $\lambda_{\text {bif }}=1.4142$

\footnotetext{
${ }^{4}$ The reader may find a detailed stability analysis, for example, in the paper by Chen 6 .
} 


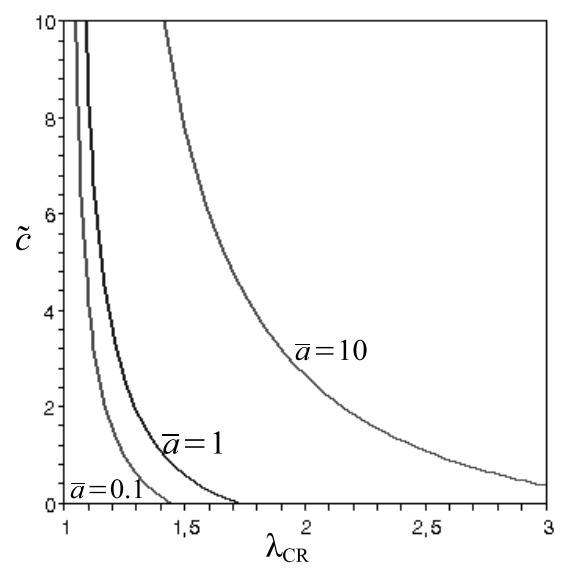

FIG. 5. Bifurcation points: Plot of Equation (25) for $\bar{a}=0.1,1$ and 10

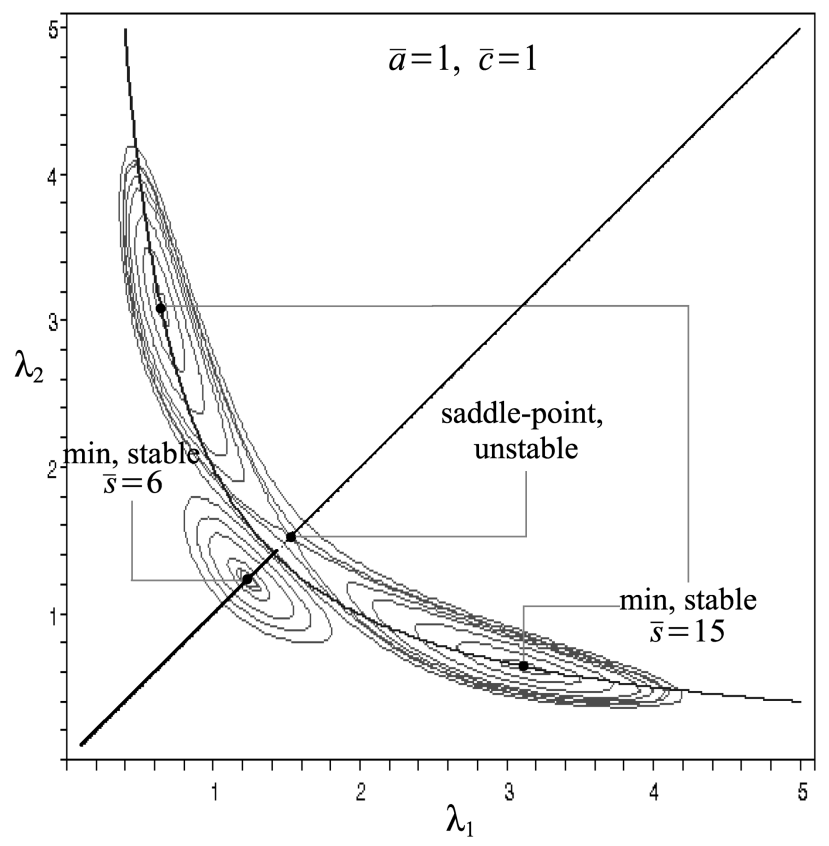

FIG. 6. Stability considerations: Plot of the specific energy $e\left(\lambda_{1}, \lambda_{2}\right)$ with $\bar{a}=1$ and $\bar{c}=1$

is arrived at. Beyond this point, the asymmetric equilibria are stable and the symmetric equilibria, as indicated by the saddle-like shape of the surface energy, become unstable. Therefore, when $s>s_{\text {bif }}=11.3137|b|$, chances of observing a deformed cylindrical body 


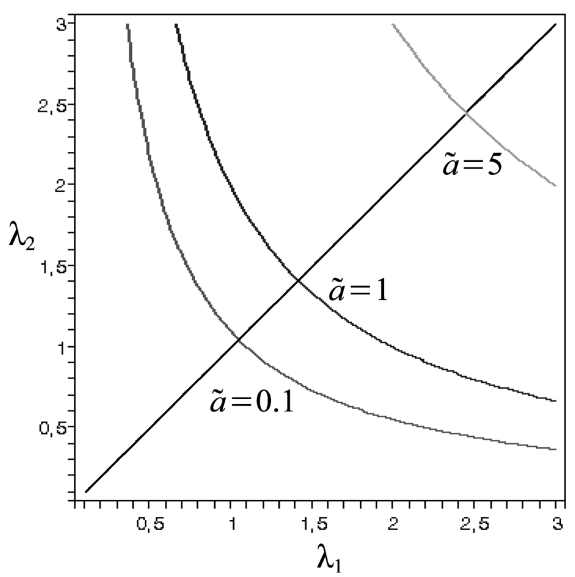

FIG. 7. Branches of asymmetric equilibria on the $\left(\lambda_{1}, \lambda_{2}\right)$-plane: Plot of Equation (26) for $\tilde{a}=0.1,1$ and 5

with square cross section practically vanish. Indeed, at any imperceptible disturbance, one of the two symmetric principal stretches increases and the other decreases. Deformation loses its symmetry and the shape of the cross section (randomly) turns into one of the two possible parallelograms, assuming a stable equilibrium configuration. It is worth noticing that this particular case of a nonlinear cylindrical body in tension shows strong qualitative analogies with Euler's beam, namely, with a slender beam in compression.

5. Application to compressible neo-Hookean materials. Setting $b=0$ in the equations of Section 4 the case of compressible neo-Hookean materials can be obtained straightforwardly. In particular, (20), (21) and (22) reduce to

$$
\begin{gathered}
\tilde{a}\left(\lambda_{1} \lambda_{2}+1\right)-\lambda_{1}^{2} \lambda_{2}^{2}+1=0, \\
\bar{s}= \pm \frac{\lambda_{1}+\lambda_{2}}{\lambda_{1} \lambda_{2}}\left\{\tilde{a}\left(\lambda_{1} \lambda_{2}-1\right)+\lambda_{1}^{2} \lambda_{2}^{2}-1\right\}, \\
\tilde{d}=2(\tilde{a}+1),
\end{gathered}
$$

where $\tilde{a}=a / c, \tilde{d}=d / c$ and $\tilde{s}=s / c$. Equation (26), for the sole material parameter $\tilde{a}$, is plotted in Figure 7. As is evident from the diagram, the asymmetric equilibrium solutions have again a hyperbola-like form, showing a qualitative trend very similar to that of compressible Mooney-Rivlin materials. Moreover, identical considerations about the stability of each single branch hold.

Finally, it is important to observe that, unlike what was shown in this section, the analogous problem, formulated for a body composed of neo-Hookean material under plane stress conditions, does not admit asymmetric solutions [8].

\section{REFERENCES}

[1] L.R.G. Treloar, Stress-strain data for vulcanized rubber under various type of deformation. Trans. Faraday Soc. 40, 59-70 (1944) 
[2] E.A. Kearsley, Asymmetric stretching of a symmetrically loaded elastic sheet. Int. J. Solid. Struct. 22, 111-119 (1986)

[3] G.P. MacSithigh, Energy-minimal finite deformations of a symmetrically loaded elastic sheet. Quart. J. Mech. Appl. Math. 39, Pt 1, 111-123 (1986) MR0827704 (87d:73036)

[4] R.W. Ogden, On the stability of asymmetric deformations of a symmetrically-tensioned elastic sheet. Int. J. Engng. Sci. 25, 1305-1314 (1987) MR0912604 (88i:73034)

[5] Y.C. Chen, Stability of homogeneous deformations of an incompressible elastic body under dead-load surface tractions. J. Elasticity 17, 223-248 (1987) MR0888317 (88d:73030)

[6] Y.C. Chen, Bifurcation and stability of homogeneous deformations of an elastic body under deadload tractions with $Z_{2}$ symmetry. J. Elasticity 25, 117-136 (1991) MR1111363 (92e:73023)

[7] H. W. Haslach Jr, Constitutive models and singularity types for an elastic biaxially loaded rubber sheet. Math. Mech. Solids 5, 41-73 (2000) MR.1740047 (2001h:74011)

[8] A. M. Tarantino, Asymmetric Equilibrium Configurations of Symmetrically Loaded Isotropic Square Membranes. J. Elasticity 69, 73-97 (2002) MR2020511(2004i:74072)

[9] R. W. Ogden, Local and global bifurcation phenomena in plane-strain finite elasticity. Int. J. Solid Struct. 21, 121-132 (1985)

[10] R. W. Ogden, On nonuniqueness in the traction boundary-value problem for a compressible elastic solid. Quart. Appl. Math. 42, 337-344 (1984) MR0757172 (85f:73058)

[11] P. G. Ciarlet, Mathematical Elasticity. Vol. I: Three-Dimensional Elasticity. North-Holland, 1988 MR.0936420 (89e:73001)

[12] R. S. Rivlin, Large elastic deformations of isotropic materials, IV. Further developments of the general theory. Phil. Trans. Roy. Soc. London A 241, 379-397 (1948) MR0027674 (10:340b)

[13] R. W. Ogden, Large deformation isotropic elasticity: On the correlation of theory and experiment for compressible rubberlike solids. Proc. Roy. Soc. London A 328, 567-583 (1972)

[14] J. M. Ball, Convexity conditions and existence theorems in nonlinear elasticity. Arch. Rational Mech. Anal. 63, 337-403 (1977) MR0475169 (57:14788)

[15] P. G. Ciarlet and G. Geymonat, Sur les lois de comportement en élasticité nonlinéaire compressible. C.R. Acad. Sci. Paris Sér. II 295, 423-426 (1982) MR0695540 (84c:73013) 